\title{
STABILITIES OF THE GENERALIZED MIXED WIDTH AND DUAL MIXED RADIAL INEQUALITIES
}

\section{HENGFANG QIU AND DEYAN ZHANG*}

Abstract. In this note, stability properties of the generalized mixed width inequality and the generalized dual mixed radial inequality are obtained in the Hausdorff distance, $L_{2}$-metric and the dual $L_{2}$-metric, respectively.

Mathematics subject classification (2010): 52A38,52A40.

Keywords and phrases: $k$-order width function, $k$-order radial function, Hausdorff distance, $L_{2}$ metric, dual $L_{2}$-metric.

\section{REFERENCES}

[1] P. R. Chernoff, An area-width inequality for convex curves, Amer. Math. Monthly, 1969, 76 (1): $34-35$.

[2] B. Fuglede, Stability in the isoperimetric problem, Bull. London Math. Soc., 1986, 18: 599-605.

[3] X. GAO, A new reverse isoperimetric inequality and its stability, Math. Inequal. Appl., 2012, 15 (3): $733-743$.

[4] L. GAO \& Y. L. WANG, Stability properties of the generalized Chernoff inequality, Math. Inequal. Appl., 2012, 15 (2): 281-287.

[5] H. Groemer, Stability properties of geometric inequalities, Amer. Math. Monthly, 1990, 97 (5): 382-394.

[6] H. Groemer \& R. SchneIder, Stability estimates for some geometric inequalities, Bull. London Math. Soc., 1991, 23: 67-74.

[7] Y. Y. MAO \& Y. L. YANG, A generalized mixed width inequality and a generalized dual mixed radial inequality, Results Math., 2019, 74: 123-134.

[8] K. OU \& S. L. Pan, Some remarks about closed convex curves, Pacific J. Math., 2010, 248 (2): 393-401.

[9] S. L. PAN \& H. P. XU, Stability of a reverse isoperimetric inequality, J. Math. Anal. Appl., 2009, 350: 348-353.

[10] R. SChneIDER, On Steiner points of convex bodies, Israel J. Math., 1971, 9: 241-249.

[11] R. SchneIder, Convex bodies: the Brunn-Minkowski theory, Second expanded edition, Cambridge University Press, Cambridge, 2014.

[12] D. Y. ZHANG \& Y. L. YANG, The dual generalized Chernoff inequality for star-shaped curves, Turkish J. Math., 2016, 40 (2): 272-282. 